\title{
Double-Balloon Endoscopy in Overt and Occult Small Bowel Bleeding: Results, Complications, and Correlation with Prior Videocapsule Endoscopy in a Tertiary Referral Center
}

\author{
Carlijn Hermans ${ }^{1}$, Arnold Stronkhorst ${ }^{1}$, Annemarie Tjhie-Wensing ${ }^{2}$, Jan Kamphuis ${ }^{3}$, Bas van Balkom ${ }^{4}$, Rob Dahlmans ${ }^{5}$ \\ and Lennard Gilissen ${ }^{1}$ \\ Department of Gastroenterology and Hepatology, ${ }^{1}$ Catharina Ziekenhuis, Eindhoven, ${ }^{2}$ Elkerliek Ziekenhuis, Helmond, ${ }^{3}$ Máxima Medisch \\ Centrum, Veldhoven, ${ }^{4}$ Bernhoven Ziekenhuis, Uden, ${ }^{5}$ Sint Jans Gasthuis, Weert, Netherlands
}

Background/Aims: Videocapsule endoscopy (VCE) and double-balloon endoscopy (DBE) allow deep exploration in patients with suspected small bowel pathology. VCE is often performed as an initial small bowel examination to explore whether an intervention by DBE is indicated and to determine insertion route. The study aim was to evaluate the correlation between DBE and VCE in patients with obscure or overt bleeding or anemia, as well as intervention frequency, and complications.

Methods: Retrospective observational study.

Results: DBE procedures $(n=205)$ showed small bowel lesions in $64 \%$ cases. Antegrade DBE showed positive results in $79 \%$ cases, mostly angiodysplasias (63\%). Retrograde DBE showed positive results in $22 \%$ cases. An intervention was performed in $64 \%$ of DBE procedures. The major complication rate was $0.5 \%$, which was one case of perforation. Pancreatitis did not occur. The overall diagnostic agreement was $66 \%$ among the 134 DBEs with preceded VCE.

Conclusions: In cases of overt or occult bleeding or anemia, DBE was positive in 64\%, with only a few complications. Positive correlation was $66 \%$ among initially performed VCEs and DBEs. Owing to the time-consuming and invasive character of DBE, performing VCE before DBE might still be clinically relevant. Clin Endosc 2017;50:69-75

Key Words: Double-balloon endoscopy; Capsule endoscopy; Anemia; Hemorrhage; Intestine, small

\section{INTRODUCTION}

The diagnosis of small bowel pathology is complicated by location, total length, and tortuous anatomy. ${ }^{1,2} \mathrm{~A}$ small number of patients with gastrointestinal complaints cannot be diagnosed by standard esophagogastroduodenoscopy and ileocolonoscopy. Videocapsule endoscopy (VCE) and double-balloon endoscopy (DBE) are techniques that allow deep-

Received: June 4, 2016 Revised: August 23, 2016

Accepted: October 6, 2016

Correspondence: Carlijn Hermans

Department of Gastroenterology and Hepatology, Catharina Ziekenhuis, Michelangelolaan 2, 5623 EJ Eindhoven, Netherlands

Tel: +31-65-053-0679, Fax: +31-40-239-9751, E-mail: Cjmhermans@gmail.com

(cc) This is an Open Access article distributed under the terms of the Creative Commons Attribution Non-Commercial License (http://creativecommons.org/ licenses/by-nc/3.0) which permits unrestricted non-commercial use, distribution, and reproduction in any medium, provided the original work is properly cited. er exploration of the small bowel and have opened the "black box" of the abdomen. ${ }^{1}$ Next to visualization, DBE also enables the gastroenterologist to perform diagnostic biopsies and a variety of therapeutic interventions.

Previous studies have shown that both VCE and DBE are mostly performed for gastrointestinal bleeding or iron deficiency anemia of unknown cause. ${ }^{2-4}$ The origin of obscure gastrointestinal bleeding is usually a benign lesion (angiodysplasia) located in the small bowel. A meta-analysis that included 642 patients has shown a diagnostic yield of $62 \%$ in VCE. DBE has shown a comparable diagnostic yield of $56 \%{ }^{5}$ VCE is often used before DBE to select patients with small bowel abnormalities ${ }^{6}$ because, compared to DBE, VCE is more patient-friendly, less invasive, and safe. In addition, VCE may provide an indication for the appropriate DBE insertion route, based on the location of the findings. However, a recently published review by Akyuz and Akyuz advised performing 
DBE after negative VCE when pathology is clinically suspected. ${ }^{7}$ Next, DBE without prior VCE seems to be a more cost-effective approach. ${ }^{8,9}$

VCE and DBE are performed in Catharina Hospital since 2007. DBE is exclusively performed at this center, whereas VCE is also performed at two other hospitals in the region of Eindhoven, the Netherlands, which refer patients for DBE in cases of clinically relevant findings, requiring an intervention (e.g., overt small bowel bleeding, iron-refractory or -dependent anemia caused by angiodysplasia, inflammation, or neoplasia). In this study, we investigated the results of DBE procedures for the indication overt or occult bleeding or anemia. A subgroup was conducted for patients who underwent videocapsule before DBE

Primary endpoints in this study were small bowel pathology detection rate of $\mathrm{DBE}$ and the diagnostic agreement with prior VCE in patients with obscure or overt bleeding. Secondary endpoints were frequency of interventions in DBE and complications related to DBE.

\section{MATERIAL AND METHODS}

\section{Patients}

This retrospective study included all patients who underwent DBE for overt or occult small bowel bleeding between September 2007 and September 2014. Exclusion criteria were DBE procedures for other indications, e.g., abdominal pain or diarrhea, DBE colonoscopy (in cases of incomplete colonoscopy), and endoscopic retrograde cholangiopancreatography with a double-balloon endoscope. Data were collected from the patient information system. Data from referred patients were collected from the referring hospitals.

Overt bleeding was defined as bleeding that is visible to the patient or clinician, e.g., hematemesis, melena, or rectal blood loss. Occult bleeding was defined as iron deficiency anemia without visible blood loss or with positive fecal occult blood test result. A focus in the small bowel was considered in all patients with negative findings on esophagogastroduodenoscopy and ileocolonoscopy.

A positive result of VCE or DBE was defined as the visualization of a small bowel lesion (e.g., erosion, ulcer, angiodysplasia, diverticulum, polyp, stenosis, or neoplasia), active bleeding without a visible lesion or vulnerable mucosa with possible tendency to bleed, and aspecific erythema. Multiple abnormalities were registered when more than one abnormality was seen in a VCE or DBE procedure.

A negative result was defined as no abnormal finding during the examination or small aspecific abnormalities, such as small red spots or visible submucosal veins. In the calcu- lation of the diagnostic agreement between VCE and DBE, a negative finding in DBE was defined as a negative result or a positive finding other than the abnormality found in VCE.

\section{DBE}

All DBE procedures were performed in one hospital (Catharina Hospital Eindhoven), using the Fujinon Double-Balloon Enteroscopy System (Fujinon GMBH, Germany) type number EN-450T5. This endoscope has a 9.4-mm outer diameter with a $2.8-\mathrm{mm}$ forceps channel diameter. Bowel cleansing consisted of 1-L polyethylene glycol solution divided into two doses to be used twice (Moviprep, Norgine Limited, United Kingdom). DBE was performed with the antegrade and/or retrograde approach, depending on the location of the abnormal finding on VCE, which was determined when the capsule detected the abnormality. When no VCE was performed before DBE, the gastroenterologists determined the insertion route based on the clinical characteristics of the complaint (e.g., color of stool), findings in previous examinations (e.g., computed tomography [CT]), and/or clinical experience. The antegrade insertion route was preferred to the retrograde insertion route because a deeper small bowel insertion can be generally achieved, ${ }^{10,11}$ and the majority of the lesions is expected to be located in the proximal to middle small bowel. ${ }^{12}$

The findings, location of findings, intervention, way of approach, and complications were carefully recorded. The location of the abnormality was determined by calculating the depth of the endoscope insertion into the small bowel: each proper step followed by adequate scope withdrawal without gliding the scope back was considered as $30 \mathrm{~cm}$. One or more interventions were performed during DBE in some patients, e.g., biopsy, argon plasma coagulation, clipping, or balloon dilatation. All DBE examinations were performed by two experienced endoscopists (LG and AS).

The DBE was performed with patients under conscious sedation, with intravenous midazolam and fentanyl or incidentally with propofol. The exact time per procedure was not reported. The range of procedure duration was 45-90 minutes and 45-75 minutes for antegrade and retrograde DBE, respectively.

\section{VCE}

VCE procedures were performed at three hospitals (Máxima Medical Centre Veldhoven, Bernhoven Uden and Catharina Hospital Eindhoven). Catharina Hospital used the Olympus videocapsule (Olympus EndoCapsule; Tokyo, Japan). The two other centers used the Pillcam videocapsule (Covidien plc, Dublin, Ireland). Both types of capsule transmit continuous video images at a rate of 2 frames/s in a $140^{\circ}$ field of view. 
Battery lifetime is at least 8 hours. Bowel cleansing for patients who underwent VCE consisted of $2 \mathrm{~L}$ polyethylene glycol solution (Moviprep, Norgine Limited, United Kingdom or Colofort, Beaufort Ipsen, France) in a single or split dose. The capsule was swallowed with one glass of water the following morning. The location of the capsule was checked 2 hours after ingestion. In cases of gastric retention of the capsule, a gastroscopy was performed to catch the capsule with Rothnet (US endoscopy, Mentor, Ohio, USA) and release it in the duodenum.

An absolute contraindication for performing VCE was a suspected or known small bowel obstruction, caused by a previous surgery, tumor, or fibrotic Crohn's disease. Relative contraindications were the presence of a pacemaker or implantable cardioverter-defibrillator and pregnancy.

\section{Statistical analysis}

Continuous variables were presented as mean with standard deviation or median with range depending on normality. Categorical variables were presented as frequency and percentage. Differences were evaluated by using the Chi-square test or Fisher's exact test in cases of small numbers. $p<0.05$ was considered statistically significant. Diagnostic agreement beyond chance was assessed by the kappa coefficient. All data were analyzed with Statistical Package for Social Science (SPSS), software version 21.0 (SPSS Inc., Chicago, IL, USA).

\section{RESULTS}

Between September 2007 and September 2014, 354 DBE procedures (mean, 50 per year) were performed. Of these, 69

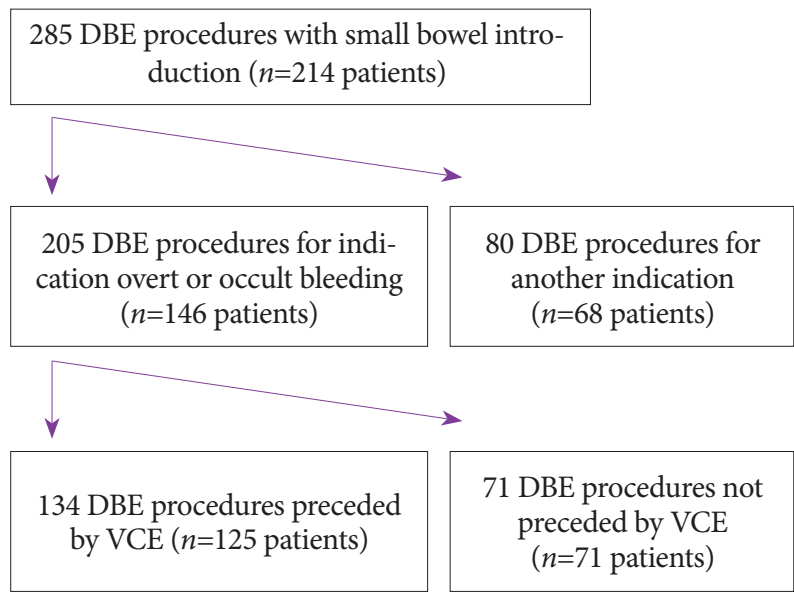

Fig. 1. Flowchart of procedures performed in our study. A total of 205 double-balloon endoscopy (DBE) procedures are performed for the indication of overt or occult bleeding, of which 134 were preceded by videocapsule endoscopy (VCE), and 71 were not preceded by VCE. were excluded from the analysis because these procedures were intended to be a colonoscopy or endoscopic retrograde cholangiopancreatography instead of small bowel visualization. Small bowel insertion during DBE occurred in 285 procedures, performed in 214 patients. In 205 procedures (in 146 patients), the indication for DBE was overt or occult bleeding or anemia. After the initial DBE, 42 patients had one or more DBEs. Some had a DBE via the same route of insertion because of recurring symptoms and some had DBE via the other route because of persisting symptoms after negative DBE procedure.

DBE for overt or occult bleeding or anemia was preceded by VCE in 134 procedures (65\%), which were performed in 125 patients. A flowchart of performed procedures is shown in Fig. 1.

This study describes the results of DBE indicated for overt or occult bleeding or anemia, with suspicion of a focus in the small bowel. The baseline characteristics of patients who underwent DBE for the indication of overt or occult bleeding or anemia are presented in Table 1.

\section{DBE}

The DBE scope was inserted antegradely in $73 \%$ of the procedures, with a mean insertion depth in the small bowel of $276 \pm 129 \mathrm{~cm}$. Retrograde insertion was performed in $27 \%$ of the procedures, with a mean depth of $79 \pm 77 \mathrm{~cm}$. None of the DBE procedures resulted in total endoscopy.

DBE procedures showed a positive finding (only small bowel lesions) in $64 \%$. Antegrade DBE procedures showed a positive result in $79 \%$, mostly angiodysplasias (63\%), as shown in Fig. 2. Retrograde DBEs were positive in $22 \%$, which showed diverse findings, as shown in Fig. 2.

Interventions were performed in $64 \%$ of DBE procedures, which consisted of 101 argon plasma coagulations, 36 diagnostic biopsies, 9 clippings, and 5 migrated stent removals.

Overall, 1 perforation ( $0.5 \%)$ occurred in all 205 DBE procedures. This perforation occurred in an anterograde DBE procedure that showed an ulcerative stenosis in the jejunum.

Table 1. Baseline Characteristics of Patients Who Underwent DBE for Indication Overt or Occult Bleeding ( $n=146$ patients).

\begin{tabular}{lc}
\hline & DBE $(\boldsymbol{n}=\mathbf{1 4 6})$ \\
\hline Age (years) (mean, min-max) & $69(18-91)$ \\
Men / women & $91 / 55$ \\
Vitamin K antagonists and NOACs & $25(17 \%)$ \\
Acetylsalicylic acid & $55(38 \%)$ \\
Clopidogrel / ticagrelor / dipyridamol & $15(10 \%)$ \\
\hline
\end{tabular}

DBE, double balloon endoscopy; NOACs, new oral anticoagulantia. 


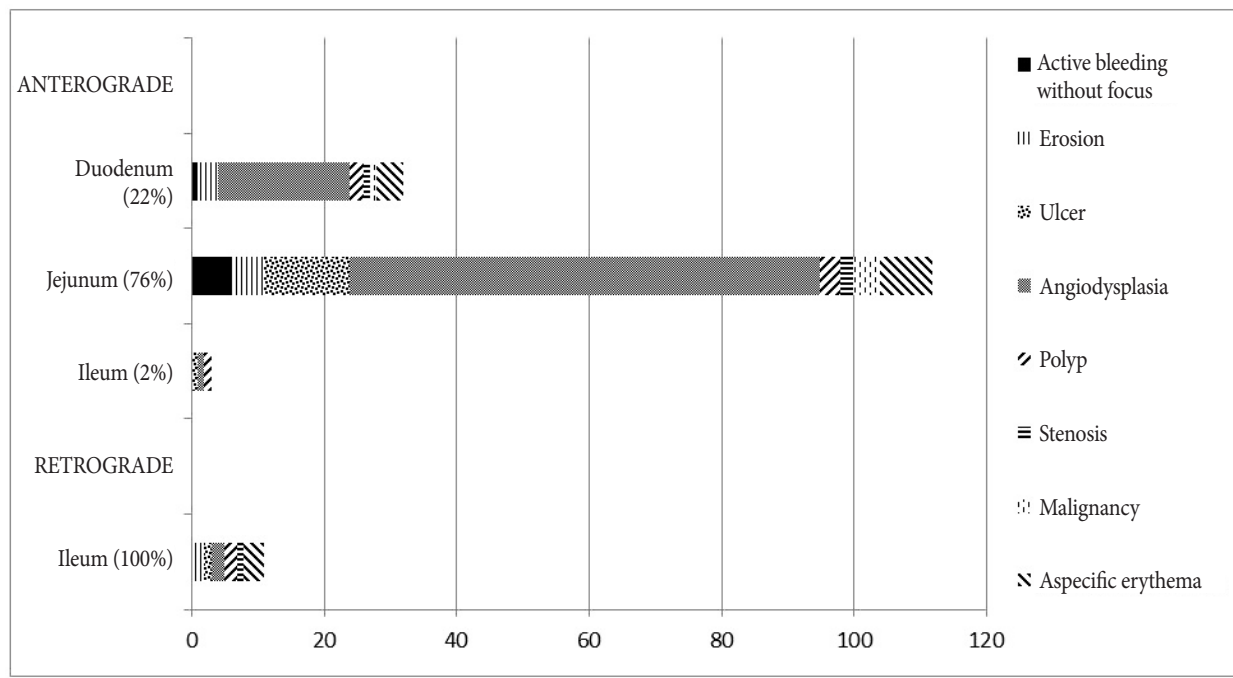

Fig. 2. Findings in antegrade $(n=149)$ and retrograde $(n=56)$ double-balloon enteroscopy for indication of overt or occult bleeding $(n=146)$ based on location. In anterograde double-balloon endoscopy (DBE) procedures, $76 \%$ of positive findings were located in the jejunum, mostly angiodysplasias (63\%). Retrograde DBEs only visualized the ileum of the small bowel and showed different findings.
The perforation occurred during diagnostic biopsy of the ulcerative stenosis, requiring wedge excision of the small bowel. In $1.5 \%$ of minor complications such as hypoxia, bradycardia, and epistaxis, these were all treated conservatively and recovered completely.

\section{VCE}

Of 205 DBE procedures, 134 were preceded by a VCE procedure (65\%). These VCE procedures showed a negative result in 7 procedures $(6 \%)$. The other procedures showed a positive result, namely 70 angiodysplasias (53\%), 35 active bleedings without visible focus (26\%), 11 ulcers (9\%), 5 erosions (4\%), 1 stenosis (1\%) and 1 aspecific erythema (1\%).

\section{Diagnostic agreement between VCE and DBE in patients with obscure or overt bleeding}

DBE was preceded by VCE in 134 patients. All 35 patients with active bleeding without visible focus on VCE underwent further examination by DBE.

In $89 \mathrm{DBE}$ procedures (66\%), the small bowel findings corresponded with small bowel findings on VCE. Of these $134 \mathrm{DBE}$ procedures, 41 had negative results, despite positive results with VCE. Of these, 19 VCEs detected angiodysplasias, 11 active bleeding without a visible focus, 5 erosions, 4 ulcers, 1 polyp, and 1 aspecific erythema. Of the three VCE negative and DBE positive procedures, the findings were erosion, terminal ileitis, and aspecific erythema.

VCEs that showed active bleeding without visible focus showed diagnostic agreement with DBE in 69\%. In these patients, DBE showed 19 angiodysplasias and 5 ulcers. The degree of diagnostic agreement between DBE and VCE based on all types of lesions is presented in Table 2.

The median time between the VCE and DBE procedures was 111 days (range, 1-1091 days). The median time between VCE with active bleeding without visible focus and DBE was 8 days (range, 1-27 days). For this subgroup and the overall group, no significant difference in the median time between VCE and DBE procedures was found between patients with consistent VCE and DBE findings and patients with different VCE and DBE findings ( $p=0.3$ ).

Overall, only a weak diagnostic agreement between VCE and DBE results was found $(\kappa=0.02)$.

\section{DISCUSSION}

This retrospective study on a large series of $\mathrm{DBE}$ procedures performed in a tertiary referral center for the indication overt or occult bleeding or anemia showed an $64 \%$ detection rate in DBE. A diagnostic agreement of $66 \%(\kappa=0.02)$ between DBE and prior VCE was found for this indication.

The DBE detection rate of $64 \%$ was similar to that of other studies (43\%-69\%). ${ }^{13-18}$ A recently published meta-analysis showed a $56 \%$ diagnostic yield in DBE. ${ }^{5}$ The most important explanation for a higher detection rate may be patient selection for DBE by performing prior VCE or radiological investigations. In most other (prospective) studies, all patients underwent both VCE and DBE, and therefore, DBE showed more negative results. Vilmann et al. ${ }^{19}$ also selected patients by using VCE and found a comparable diagnostic yield (83\%). Another possible explanation may be the definition of a "positive procedure". Some aspecific findings, mostly erythema, were classified as a positive procedure in our study. In addition, a likely referral bias in our study may play a role, as our department serves as a tertiary care referral center for small bowel examinations. Therefore, patients with a very strongly 
Table 2. Diagnostic Agreement (\%) DBE with Prior VCE for Indication Overt or Occult Bleeding according to VCE Finding

\begin{tabular}{lc}
\hline & $\begin{array}{c}\text { DBE with prior VCE } \\
(\boldsymbol{n}=134 \text { procedures })\end{array}$ \\
\hline All findings & $66(\kappa=0.02)$ \\
Active bleeding & $69(n=35)$ \\
Angiodysplasia & $75(n=74)$ \\
Erosion & $0(n=5)$ \\
Ulcer & $64(n=11)$ \\
Stenosis & $100(n=1)$ \\
Aspecific erythema & $0(n=1)$ \\
\hline
\end{tabular}

DBE, double balloon endoscopy; VCE, videocapsule endoscopy.

suspected small bowel disease are conceivably referred for DBE, which increases the chance of a positive result.

In our study, angiodysplasia was by far the most frequently found abnormality in the small bowel in DBE. This is in line with recent literature. ${ }^{20-23}$ However, other studies ${ }^{2,24}$ stated that inflammatory lesions were more frequently found compared with vascular abnormalities. This difference can be explained by the observed study populations: angiodysplasias were mostly found in Western populations, and inflammatory lesions were the most common in Eastern populations. ${ }^{2,23}$ Our study showed that most small bowel lesions are located in the proximal part of the small bowel, which is also in accordance with previous literature. ${ }^{8,23}$

In this study, $134 \mathrm{DBE}$ procedures were preceded by VCE. A negative VCE in our department is believed to make the invasive and time-consuming DBE unnecessary. This is based on the opinion that re-bleeding rates and need for transfusions were low after normal VCE. ${ }^{25}$ Prior VCE may also help in choosing the DBE insertion route. Nakamura et al. stated that the transit time and the position of the small bowel lesion were strongly related. ${ }^{26}$ In our study, a diagnostic agreement of 66\% between VCE findings and DBE was found, but with a kappa value of only 0.02 . Other prospective studies ${ }^{15,16}$ reported higher $d$ values of 0.46 to 0.76 . An explanation for this difference may be the retrospective design of our study, wherein a negative VCE was rarely followed by a DBE (seven procedures in our study). Only in cases of recurrent complaints of bleeding/anemia was DBE performed after negative VCE, in contrast to some prospective studies, wherein all patients underwent both VCE and DBE. The performance of DBE after negative VCE in cases of persistent clinical suspicion is in line with the advice in the recently published review by Akyuz and Akyuz.

In the calculation of the diagnostic agreement, 45 of 134 DBE procedures were negative in our study. However, a pos- itive finding in the DBE procedure following VCE was found in 23 procedures (51\%), but is not consistent with the VCE finding.

An explanation for the low diagnostic agreement may be spontaneous healing of lesions between VCE and DBE performance. Previous studies in patients with gastrointestinal bleeding showed a diagnostic yield of $91 \%$ if performed within 2 weeks after the initial bleeding compared with $34 \%$ in patients undergoing VCE thereafter. ${ }^{27,28}$ However, our study showed no significant difference in the time interval between VCE and DBE in consistent versus non-consistent findings.

Some remarks on complications in this study should be made. The major complication rate was $0.5 \%$ in all performed DBE procedures, which was comparable to previous studies. ${ }^{2,3,24,29,30}$ This consisted of one perforation caused by balloon dilatation of an ulcerative stenosis due to previous radiotherapy, which was successfully treated with surgical wedge excision. Acute pancreatitis was a relatively frequently occurring major complication in some other studies. ${ }^{3,24}$ Acute pancreatitis did not occur in our study probably because balloon inflation is only performed when the ligament of Treitz has been passed. Some minor complications occurred, but were believed to be a result of sedation mostly and not DBE related (bradycardia [0.6\%], dysrhythmia [0.3\%], hypoxia [0.3\%], and epistaxis [0.3\%]). All minor complications could be treated conservatively with total recovery.

Overall, DBE may be stated to be a relatively safe procedure with a minimal risk for major complications, despite the invasive character of this examination.

The participating hospitals use VCE for initial small bowel evaluation to determine whether a small bowel lesion was found and to estimate its location, unless acute overt bleeding occurred. The benefits of the VCE are the non-invasiveness and safety of the procedure and the possibility of visualization of the entire small bowel. ${ }^{11,13,31,32}$ The major benefit of DBE is the possibility to perform diagnostic biopsies and interventions compared with VCE. ${ }^{2,4,13,33}$ The disadvantages of DBE are invasiveness, sedation that is required, and the examination is labor-intensive. ${ }^{11,13,34}$ In 262 VCE procedures, 184 procedures $(70 \%)$ were not followed by a DBE, and thereby, reducing the need for a DBE procedure. In addition, our study showed that up to $34 \%$ of the DBE procedures preceded by a VCE did not find the lesion detected by VCE. However, in two cost-effectiveness analyses, ${ }^{8,9}$ that compared VCE (possibly followed by DBE) and immediate DBE for obscure bleeding, DBE without prior VCE was more cost-effective. Currently, whether performing an initial VCE before DBE is necessary for the indication overt or occult bleeding or anemia is unclear. This can be another subject of a prospective randomized trial. Special attention for costs, strain on the 
patient, and logistics of the endoscopy unit are required to examine the complete clinical effect.

Limitations of this study are the retrospective characteristics of the study, and the fact that VCE procedures were performed in three centers.

In conclusion, DBE showed a positive result in $64 \%$ of procedures in cases of overt or occult bleeding or anemia, with only a few complications. The correlation between initially performed VCE and DBE was $66 \%$, but with a relatively low kappa of 0.02 and in a retrospective setting. Significant active or occult blood loss or iron refractory disease is an indication for small bowel examination after negative gastro- and colonoscopy. Based on our and previous findings, performing VCE before DBE is recommended to determine the appropriate DBE insertion route. DBE seems to be the most helpful procedure in patients with angiodysplasia, active blood loss without clear focus, ulcer, and stenosis in VCE.

\section{Conflicts of Interest}

The authors have no financial conflicts of interest.

\section{REFERENCES}

1. Aktas H, Mensink PB. Small bowel diagnostics: current place of small bowel endoscopy. Best Pract Res Clin Gastroenterol 2012;26:209-220.

2. Xin L, Liao Z, Jiang YP, Li ZS. Indications, detectability, positive findings, total enteroscopy, and complications of diagnostic double-balloon endoscopy: a systematic review of data over the first decade of use. Gastrointest Endosc 2011;74:563-570.

3. Möschler O, May A, Müller MK, Ell C. Complications in and performance of double-balloon enteroscopy (DBE): results from a large prospective DBE database in Germany. Endoscopy 2011;43:484-489.

4. Kondo J, lijima H, Abe T, et al. Roles of double-balloon endoscopy in the diagnosis and treatment of Crohn's disease: a multicenter experience. J Gastroenterol 2010;45:713-720.

5. Teshima CW, Kuipers EJ, van Zanten SV, Mensink PB. Double balloon enteroscopy and capsule endoscopy for obscure gastrointestinal bleeding: an updated meta-analysis. J Gastroenterol Hepatol 2011;26:796-801.

6. Gay G, Delvaux M, Fassler I. Outcome of capsule endoscopy in determining indication and route for push-and-pull enteroscopy. Endoscopy 2006;38:49-58.

7. Akyuz U, Akyuz F. Diagnostic and therapeutic capability of double-balloon enteroscopy in clinical practice. Clin Endosc 2016;49:157-160.

8. Gerson L, Kamal A. Cost-effectiveness analysis of management strategies for obscure GI bleeding. Gastrointest Endosc 2008;68:920-936.

9. Somsouk M, Gralnek IM, Inadomi JM. Management of obscure occult gastrointestinal bleeding: a cost-minimization analysis. Clin Gastroenterol Hepatol 2008;6:661-670.

10. Sanaka MR, Navaneethan U, Kosuru B, Yerneni H, Lopez R, Vargo JJ. Antegrade is more effective than retrograde enteroscopy for evaluation and management of suspected small-bowel disease. Clin Gastroenterol Hepatol 2012;10:910-916.

11. Westerhof J, Weersma RK, Koornstra JJ. Investigating obscure gastrointestinal bleeding: capsule endoscopy or double balloon enteroscopy? Neth J Med 2009;67:260-265.

12. Heine GD, Hadithi M, Groenen MJ, Kuipers EJ, Jacobs MA, Mulder CJ. Double-balloon enteroscopy: indications, diagnostic yield, and compli- cations in a series of 275 patients with suspected small-bowel disease. Endoscopy 2006;38:42-48.

13. Tae $\mathrm{CH}$, Shim KN. Should capsule endoscopy be the first test for every obscure gastrointestinal bleeding? Clin Endosc 2014;47:409-414.

14. Arakawa D, Ohmiya N, Nakamura M, et al. Outcome after enteroscopy for patients with obscure GI bleeding: diagnostic comparison between double-balloon endoscopy and videocapsule endoscopy. Gastrointest Endosc 2009;69:866-874.

15. Fukumoto A, Tanaka S, Shishido T, Takemura Y, Oka S, Chayama K. Comparison of detectability of small-bowel lesions between capsule endoscopy and double-balloon endoscopy for patients with suspected small-bowel disease. Gastrointest Endosc 2009;69:857-865.

16. Marmo R, Rotondano G, Casetti T, et al. Degree of concordance between double-balloon enteroscopy and capsule endoscopy in obscure gastrointestinal bleeding: a multicenter study. Endoscopy 2009;41:587592.

17. Nakamura M, Niwa Y, Ohmiya N, et al. Preliminary comparison of capsule endoscopy and double-balloon enteroscopy in patients with suspected small-bowel bleeding. Endoscopy 2006;38:59-66.

18. Tenembaum D, Sison C, Rubin M. Accuracy of community based video capsule endoscopy in patients undergoing follow up double balloon enteroscopy. World J Gastrointest Endosc 2013;5:154-159.

19. Vilmann P, Jensen T, Hendel J. Double balloon endoscopy in obscure GI bleeding: the Danish experience. Gastrointest Endosc 2007;66(3 Suppl):S63-S65.

20. Gerson LB, Batenic MA, Newsom SL, Ross A, Semrad CE. Long-term outcomes after double-balloon enteroscopy for obscure gastrointestinal bleeding. Clin Gastroenterol Hepatol 2009;7:664-669.

21. Carey EJ, Leighton JA, Heigh RI, et al. A single-center experience of 260 consecutive patients undergoing capsule endoscopy for obscure gastrointestinal bleeding. Am J Gastroenterol 2007;102:89-95.

22. Cellier C. Obscure gastrointestinal bleeding: role of videocapsule and double-balloon enteroscopy. Best Pract Res Clin Gastroenterol 2008;22:329-340.

23. Bollinger E, Raines D, Saitta P. Distribution of bleeding gastrointestinal angioectasias in a Western population. World J Gastroenterol 2012;18:6235-6239.

24. Ohmiya N, Yano T, Yamamoto H, et al. Diagnosis and treatment of obscure GI bleeding at double balloon endoscopy. Gastrointest Endosc 2007;66(3 Suppl):S72-S77.

25. Lai LH, Wong GL, Chow DK, Lau JY, Sung JJ, Leung WK. Long-term follow-up of patients with obscure gastrointestinal bleeding after negative capsule endoscopy. Am J Gastroenterol 2006;101:1224-1228.

26. Nakamura M, Ohmiya N, Shirai O, et al. Route selection for double-balloon endoscopy, based on capsule transit time, in obscure gastrointestinal bleeding. J Gastroenterol 2010;45:592-599.

27. Bresci G, Parisi G, Bertoni M, Tumino E, Capria A. The role of video capsule endoscopy for evaluating obscure gastrointestinal bleeding: usefulness of early use. J Gastroenterol 2005;40:256-259.

28. Hartmann D, Schmidt H, Bolz G, et al. A prospective two-center study comparing wireless capsule endoscopy with intraoperative enteroscopy in patients with obscure GI bleeding. Gastrointest Endosc 2005;61:826832.

29. Mensink PB, Haringsma J, Kucharzik T, et al. Complications of double balloon enteroscopy: a multicenter survey. Endoscopy 2007;39:613-615.

30. Gerson LB, Tokar J, Chiorean M, et al. Complications associated with double balloon enteroscopy at nine US centers. Clin Gastroenterol Hepatol 2009;7:1177-1182.e3.

31. Milano A, Balatsinou C, Filippone A, et al. A prospective evaluation of iron deficiency anemia in the GI endoscopy setting: role of standard endoscopy, videocapsule endoscopy, and CT-enteroclysis. Gastrointest Endosc 2011;73:1002-1008.

32. Kopylov U, Seidman EG. Role of capsule endoscopy in inflammatory bowel disease. World J Gastroenterol 2014;20:1155-1164.

33. Akarsu M, Akkaya Özdinç S, Celtik A, Akpınar H. Diagnostic and 
Hermans C et al. Double-Balloon Endoscopy in Overt or Occult Small Bowel Bleeding

therapeutic efficacy of double-balloon endoscopy in patients with small intestinal diseases: single-center experience in 513 procedures. Turk J Gastroenterol 2014;25:374-380.
34. Hussan H, Crews NR, Geremakis CM, Bahna S, LaBundy JL, Hachem C. Predictors of double balloon endoscopy outcomes in the evaluation of gastrointestinal bleeding. World J Gastrointest Endosc 2014;6:248-253. 